\title{
Spinal Cord Injury due to Cervical Disc Herniation Caused by Bench Pressing
}

\section{Futoshi Suetsuna ${ }^{1,2 *}$, Yoshihiko Okudera1, Toshihiro Tanaka ${ }^{1}$ and Takuya Tamura ${ }^{2}$}

${ }^{1}$ Department of Orthopaedic Surgery, Hachinohe Municipal Hospital, Japan

${ }^{2}$ Department of Rehabilitation, Hachinohe Municipal Hospital, Hachinohe, Japan

\begin{abstract}
The authors report the case of a 24-year-old man who presented with sudden onset quadriplegia while doing a bench press in the supine position. He had a history of cervical disc herniation at the C6/7 level. Plain x-rays showed no spinal fracture and no spinal canal stenosis. MRI revealed a C6/7 disc herniation that severely compressed the spinal cord. Physical exam showed complete spinal cord injury below C8 level. Urgent C6/7 anterior decompression and fusion using hydroxyapatite (HA) was performed. A large, sequestrated herniation mass into the spinal canal was removed. His neurological deficits improved gradually after surgery. Over 3 years postoperatively, he can walk by himself without crutches, though he has slight motor weakness of left finger and foot, paresthesia below C8 on the right, and slight bladder disturbance.

Many cases of cervical spinal cord injury caused by sporting injury have been reported. Those cases generally accompanied high energy trauma such as fracture dislocations. This case did not involve any large cervical motion before injury or any spinal fracture. This very important case shows the possibility of a spinal cord injury in supine position without high energy trauma. Patients with cervical disc herniation should be counseled about the risk of participating in sports, including those without significant neck motion.
\end{abstract}

Keywords: Spinal cord injury; Cervical spine; Disc herniation; Bench press

Abbreviations: SCI: Spinal Cord Injury; CT: Computerized Tomography; HA: Hydroxyapatite; MRI: Magnetic Resonance Imaging; BTR: Biceps Tendon Reflex; TTR: Triceps Tendon Reflex; PTR: Patellar Tendon Reflex; ATR: Achilles Tendon Reflex

\section{Introduction}

Many cases with spinal cord injuries caused by sports injury have been reported. All of those cases have involved high energy trauma. These injuries are typically the result of an external force, such as a direct blow to the head and subsequent forced hyperextension or hyperflexion of the cervical spine. Previous reports have involved high speed or contact sports like snowboarding, skiing, and rugby. However, cervical spinal cord injuries rarely happen in the absence of violent motion of the cervical spine or external high-energy force to the cervical spine. We are not aware of any previously published reports of spinal cord injury due to bench pressing. In this study, we report the case of a 24-year-old man with a complete cervical spinal cord injury caused by bench pressing.

\section{Case Report}

\section{History and presentation}

This 24-year-old man had a 3-year history of cervical disc herniation at the C6/7 level. He was evaluated at another hospital in 2000 because he had felt neck pain and numbness of his fingers about twice a year. So, MRI was done and C6/7 disc herniation was diagnosed. However, his complaints quickly improved and then he paid no attention to his cervical disc herniation. In April 2003, while performing a bench press in supine position with $90 \mathrm{~kg}$, he suddenly became paralyzed and was taken to the hospital. Once a spinal cord injury was diagnosed, he was transferred to our hospital. High-dose methylprednisolone therapy was started within 6 hours of injury.

\section{Neurological examination}

Deep tendon reflexes (BTR and TTR) of upper extremities were normal. Pathologic reflexes (Hoffman, Wartenberg and Babinski) were negative. Deep tendon reflexes (PTR and ATR) of left lower extremity were negative; the right PTR was negative and right ATR was positive. Bulbocavernous reflex was negative. He showed Grade 0 muscle weakness of bilateral legs and below finger flexion. Anesthesia below C8 dermatome was present in bilateral upper and lower extremities.

\section{Radiographic examination}

Plain radiographs of the cervical spine revealed no spinal canal stenosis and no fracture dislocations (Figure 1). T1- and T2- weighted MR images showed cervical disc herniation that severely compressed the spinal cord at C6/7 level and no bleeding of the retropharyngeal space (Figure 2 left and right).

When the patient was initially evaluated in 2000, MRI showed C6/7 disc herniation without high signal intensity (Figure 3 left and right). 6 days prior to the bench press injury in 2003, a follow-up evaluation for the cervical disc herniation was completed. Flexion and extension radiographs of the cervical spine showed no instability and no spinal canal stenosis. In addition, MRI revealed that the $\mathrm{C} 6 / 7$ disc herniation had increased in size in comparison with that in 2000 (Figure 4 left and right).

\section{Operation}

Urgent surgery was performed. The patient was placed supine. Left transverse incision at C6/7 level was done. On exposing the C6 and 7 vertebral bodies, we observed no disruption of anterior longitudinal

*Corresponding author: Futoshi Suetsuna, Department of Orthopaedic Surgery \& Rehabilitation, Hachinohe Municipal Hospital, 1, Bishamondaira, Tamukai Hachinohe, Aomori, 031-0011, Japan, Tel: +81-178-72-5111; Fax: +81-178-725185; E-mail: drsue@hospital.hachinohe.aomori.jp

Received December 27, 2013; Accepted January 24, 2014; Published January 27,2014

Citation: Suetsuna F, Okudera Y, Tanaka T, Tamura T (2014) Spinal Cord Injury due to Cervical Disc Herniation Caused by Bench Pressing. J Spine 3: 154 doi:10.4172/2165-7939.1000154

Copyright: (c 2014 Suetsuna F, et al. This is an open-access article distributed under the terms of the Creative Commons Attribution License, which permits unrestricted use, distribution, and reproduction in any medium, provided the original author and source are credited. 
Citation: Suetsuna F, Okudera Y, Tanaka T, Tamura T (2014) Spinal Cord Injury due to Cervical Disc Herniation Caused by Bench Pressing. J Spine 3: 154. doi:10.4172/2165-7939.1000154

Page 2 of 4

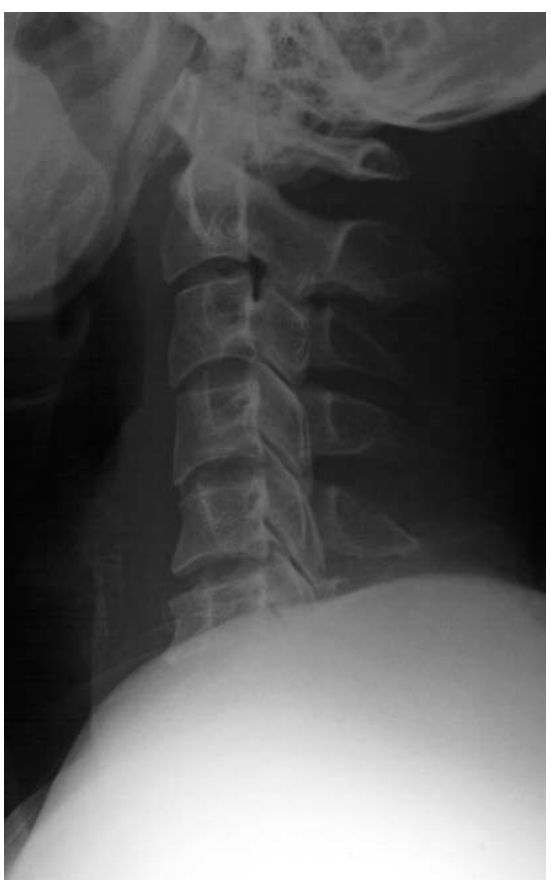

Figure 1. Preoperative radiograph of the cervical spine, revealing no spinal canal stenosis and no fracture dislocations.
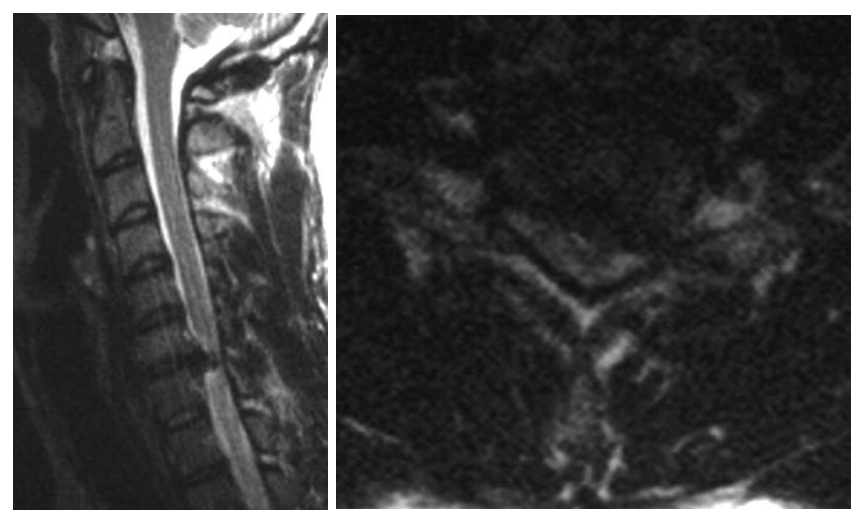

Figure 2. Preoperative MR images. Left: sagittal T2-weighted MR image showing severely compressed spinal cord at C6/7 level.

Right: axial T2-weighted MR image showing cervical disc herniation at C6/7 level.

ligament. We resected the inferior portion of C6 and superior portion of C7 with an air drill to make a rectangular trough and decompress larger than the compressed areas of the cord. There were large sequestrated herniations accompanied with end plates in the epidural space. After removing the sequestrated herniation mass, hydroxyapatite (height; 9 $\mathrm{mm}$, width; $15 \mathrm{~mm}$, depth; $12 \mathrm{~mm}$ ) was countersunk into the trough without use of iliac crest bone graft [1]. Operation time was 62 minutes and blood loss was $5 \mathrm{ml}$. No dural tear was seen.

\section{Postoperative course}

The patient was treated postoperatively with an external Philadelphia collar. Postoperative plain cervical radiographs showed C6/7 anterior fusion using hydroxyapatite (Figure 5 left and right). Radiographs of the cervical spine one year and 3 years after surgery showed no radiolucent zone around the HA and no failure of the HA, respectively (Figure 6 left). Computed tomograms revealed satisfactory bone healing to the HA (Figure 6 right).
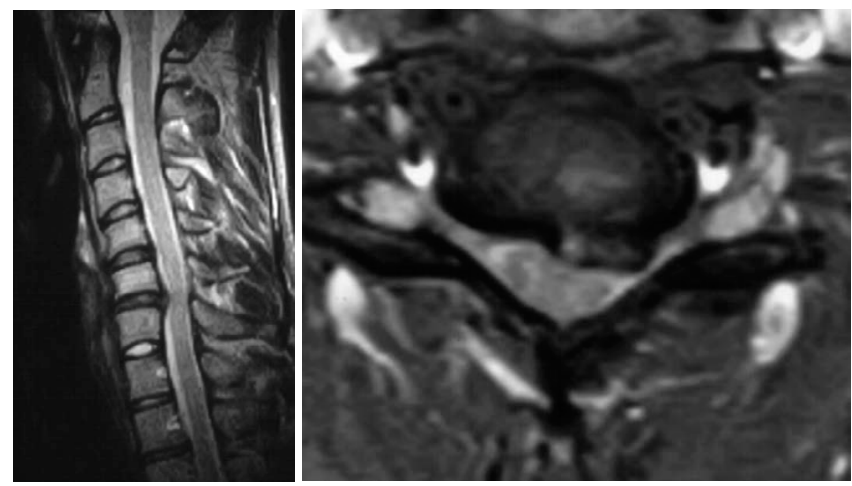

Figure 3. MR images in 2000. Left: sagittal T2-weighted MR image showing C6/7 disc herniation without high signal intensity.

Right: axial T1-weighted MR image showing C6/7 herniation compressing from central and left side.
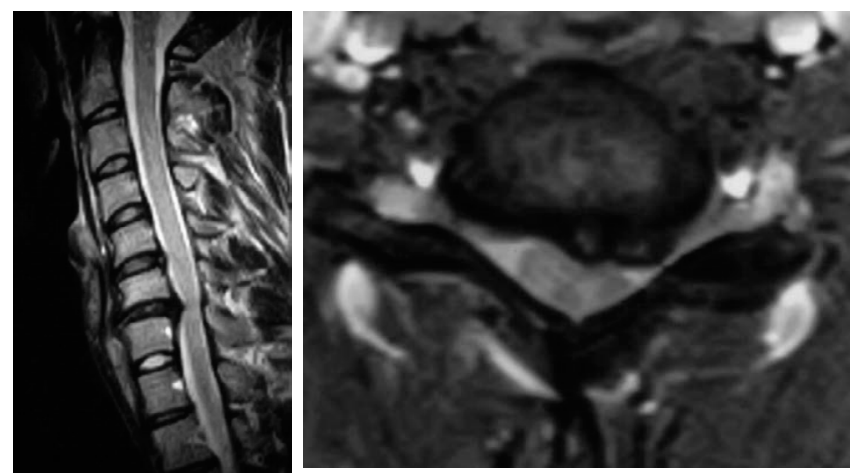

Figure 4. MR images obtained 6 days before injury. Sagittal (left) and axial (right) T2-weight MR image showing C6/7 herniation increased in size in comparison with that in Figure 3.
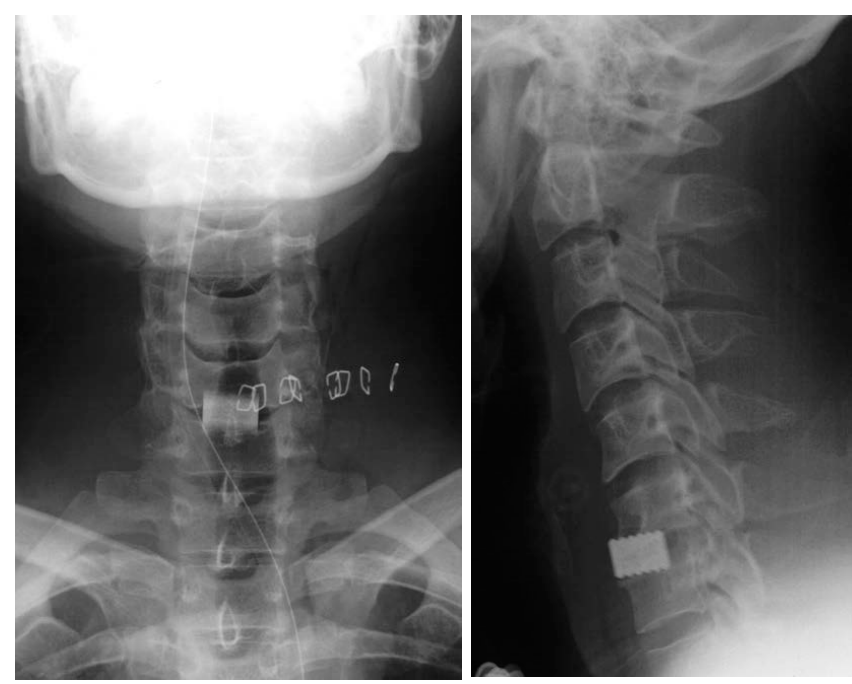

Figure 5. Postoperative radiographs. anterior-posterior view (left) and lateral view (right), showing anterior fusion using $\mathrm{HA}$. 


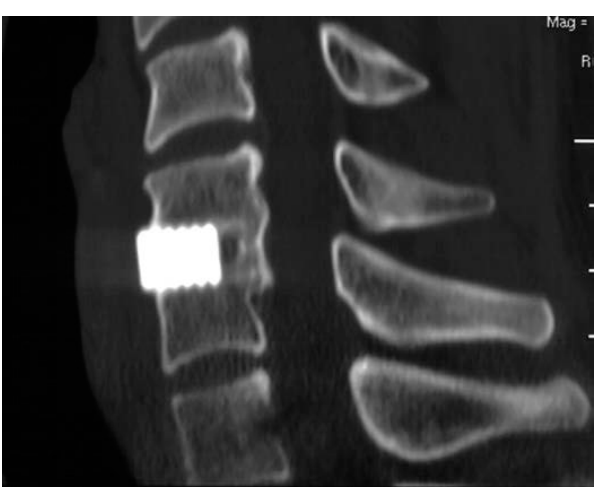

Figure 6. Lateral radiograph three year after surgery (left) and 3DCT (right) 2 year after surgery showing solid fusion without no radiolucent zone around the HA.

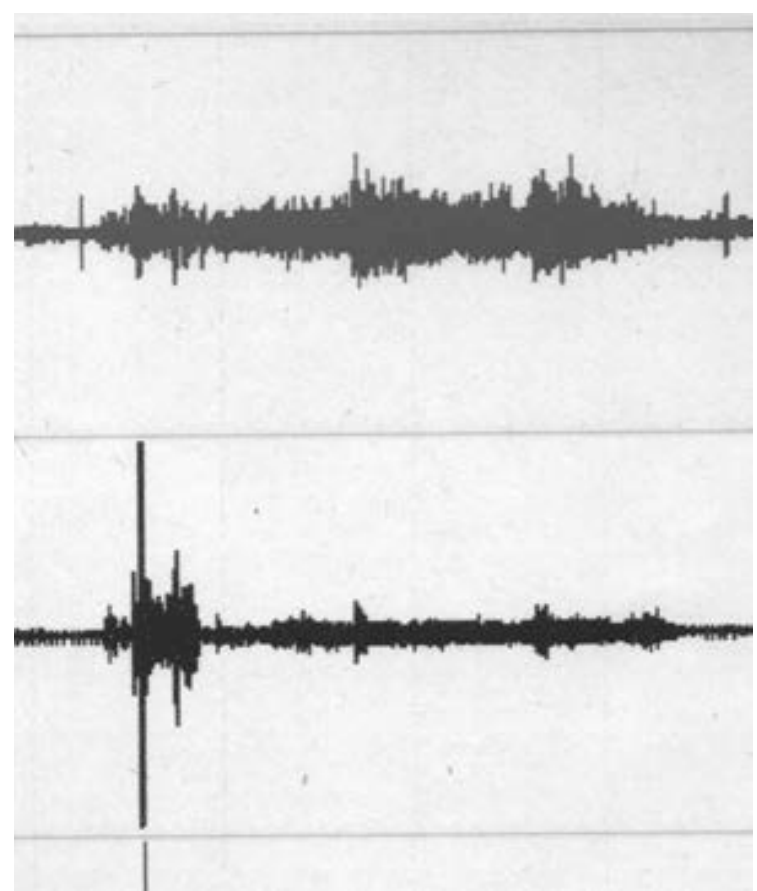

Figure 7. Superficial electromyography of the neck flexor and neck extensor muscles. EMGs of neck flexor muscles (upper) and neck extensor muscles (lower) while performing a bench press in supine position with $60 \mathrm{Kg}$. It shows continuous muscle contractions during lifting.

His neurological palsy gradually improved postoperatively. 3 years after surgery, he could walk without crutches. Manual motor testing of the upper and lower extremities, showed Grade 5 (normal) of right finger flexion and right leg and Grade 3 muscle weakness of left finger flexion and Grade 2 to 4 muscle weakness of the left leg. He also had hypesthesia of touch sensation below right $\mathrm{C} 8$ dermatome.

\section{Discussion}

Almost all cervical spinal cord injuries are a result of severe traumatic accidents such as traffic accidents, labor accidents or sport accidents [2]. Of those SCI, most accompany fractures and/ or dislocations of the cervical spine. There have been many reports of cervical SCI without spinal fracture. In those cases, cervical SCI are caused by low-energy trauma, like falls from standing height in the elderly. Many factors have been identified as risk factors for SCI without spinal fractures; spondylotic cervical spinal canal stenosis [35] and ossification of posterior longitudinal ligaments are common examples. The mechanism of those injuries is mainly hyperextension of the cervical spine [6]. So, the pathology of those injuries is widely recognized as a central spinal cord injury [7].

The rate of traumatic cervical disc herniation accompanied by spinal injury using myelography and CT was low $[8,9]$. But, recently, MRI has made it possible to clearly identify traumatic cervical disc herniations associated with spinal fractures $[10,11]$. Surgical outcomes are strongly influenced by removal of the sequestrated herniated mass as well as the reduction and fixation of the cervical fractures. In addition, the incidence of herniated nucleus pulposus was seen even in patients without spinal fractures [11]. But, there is no report on cervical SCI without trauma such as hyperextension or flexion of the cervical spine.

This case occurred while a patient with a history of cervical disc herniation was bench pressing $90 \mathrm{~kg}$ in supine position. Why did the disc herniation produce acute cervical SCI? Bench press exercise mainly strengthens the pectoral major muscle, shoulder muscles, biceps and triceps muscle; it involves no strong neck extension or flexion motion. One may not expect significant cervical muscle activity or injury to the cervical spine. We examined the superficial electromyography of the circumferential muscles of the neck during bench press exercise. As the weight is lifted, there are muscle contractions of the extensor and flexor muscles of the neck (Figure 7). There are eccentric contractions in the anterior flexor muscles and concentric contractions in the posterior extensor muscles of the neck. Generally, the anterior flexor muscles act on the anterior bending of the cervical spine. On the other hand, the posterior extensor muscles act on the posterior inclination of the head and neck. In addition, when lifting by bench press, the players shut their teeth and do not raise their mandibles. So, during the bench press, contraction of the anterior flexor muscles increase further. Therefore, even if there is hardly movement of the cervical spine, powers of posterior inclination of the head and anterior bending of the neck produce an axis force for cervical intervertebral discs. Therefore, bench press activity may exacerbate a pre-existing disc herniation. This case is a very important case for patients with cervical disc herniation, even if it is asymptomatic, who are involved in sports. Torg et al. [12] reported that $34 \%$ of athletes with a history of transient quadriplegia had findings of cervical cord compression in MR imaging. So, we think that the existence of cervical disc herniation will be one of the risk factors for spinal cord injury. Our experience suggests that serious cervical spinal palsy might occur in spite of no apparent cervical motion and noncontact sports. Physicians should be aware of the risks of activities such as the bench press in patients with cervical disc herniation.-

\section{Conclusion}

The possibility of cervical spinal cord injury during bench press exercises should be considered in patients with cervical disc herniation. Patients with cervical disc herniation should be counseled about the risks of participating in sports, including those without significant neck motion.

\section{Disclaimer}

None of the authors involved in this study has a financial interest or relationship with the manufacturer or any device used.

\section{References}

1. Suetsuna F, Yokoyama T, Kenuka E, Harata S (2001) Anterior cervical fusion using porous hydroxyapatite ceramics for cervical disc herniation. a two-year follow-up. Spine J 1: 348-357. 
2. Cantu RC, Mueller FO (2003) Catastrophic spine injuries in American football, 1977-2001. Neurosurgery 53: 358-362.

3. Pavlov H, Torg JS, Robie B, Jahre C (1987) Cervical spinal stenosis: determination with vertebral body ratio method. Radiology 164: 771-775.

4. Torg JS (1990) Cervical spinal stenosis with cord neurapraxia and transient quadriplegia. Clin Sports Med 9: 279-296.

5. Torg JS, Naranja RJ Jr, Pavlov H, Galinat BJ, Warren R, et al. (1996) The relationship of developmental narrowing of the cervical spinal canal to reversible and irreversible injury of the cervical spinal cord in football players. $J$ Bone Joint Surg Am 78: 1308-1314.

6. Taylor AR (1948) The mechanism of injury to the spinal cord in the neck without damage to the vertebral column. J Bone Joint Surg (Br) 30: 245-248.

7. Schneider RC, Cherry G, Pantek H (1954) The syndrome of acute centra cervical spinal cord injury; with special reference to the mechanisms involved in hyperextension injuries of cervical spine. J Neurosurg 11: 546-577.
8. Allen RL, Perot PL Jr, Gudeman SK (1985) Evaluation of acute nonpenetrating cervical spinal cord injuries with CT metrizamide myelography. J Neurosurg 63: $510-520$.

9. Cooper PR, Cohen W (1984) Evaluation of cervical spinal cord injuries with metrizamide myelography-CT scanning. J Neurosurg 61: 281-289.

10. Harrington JF, Likavec MJ, Smith AS (1991) Disc herniation in cervical fracture subluxation. Neurosurgery 29: 374-379.

11. Rizzolo SJ, Piazza MR, Cotler JM, Balderston RA, Schaefer D, et al. (1991) Intervertebral disc injury complicating cervical spine trauma. Spine (Phila Pa 1976) 16: S187-189.

12. Torg JS, Corcoran TA, Thibault LE, Pavlov H, Sennett BJ, et al. (1997) Cervical cord neurapraxia: classification, pathomechanics, morbidity, and management guidelines. J Neurosurg 87: 843-850. 